\title{
Gazettes sous influence : le Courrier du Bas-Rhin, la Gazette des Deux-Ponts et les sujets touchant la Russie vers 1770
}

\author{
Georges Dulac \\ Institut de recherche sur la Renaissance, l'Âge classique et les Lumières \\ (IRCL, CNRS et Université Montpellier 3) \\ gedulac@wanadoo.fr
}

\begin{abstract}
Résumé
Vers 1770, la campagne anti-russe du gouvernement français tend à dégrader l'image de la Russie, ce qui lèse gravement ses intérêts, notamment quand elle doit emprunter pour financer la guerre avec la Turquie. Aussi le prince Dmitri Alexeevitch Golitsyn, ministre plénipotentiaire à La Haye, se montre-t-il alors très actif sur ce terrain. Sa correspondance avec Pétersbourg témoigne de ses efforts, aux effets inégaux, pour influer sur les informations diffusées par quelques journaux: principalement le Courrier du Bas-Rhin, publié par Jean Manzon à Trèves, sous contrôle prussien, et dans une moindre mesure les deux Gazettes des Deux-Ponts, l'une politique, l'autre littéraire. Le journaliste de Trèves, qui trouve son intérêt à prendre le parti de la Russie, met en ouvre en sa faveur un discours journalistique abondant et parfois très élaboré. Cependant, la ligne du journal subit des fluctuations sensibles, selon l'évolution de la situation et à la suite de diverses interventions, dont celles du roi de Prusse et d'autre part de Stanislas-Auguste, qui pensionne un temps le journaliste. La Gazette des deux Ponts pratique l'information orientée avec plus de finesse, et, comme la gazette littéraire, accorde une large place à la matière russe: mais sur le plan politique, son traitement reste le plus souvent sous influence française et répond rarement aux vœux de D. A. Golitsyn.
\end{abstract}

\section{Mots clé}

presse francophone en Allemagne; le Courrier du Bas-Rhin; la Gazette des Deux-Ponts; Russie ; diplomatie russe ; influence sur la presse

Le 27 avril 1770, quelques semaines après avoir pris ses fonctions de ministre plénipotentiaire à La Haye, le prince Dmitri Alekseevitch Golitsyn transmet à Pétersbourg une lettre signée de «l'auteur de la Gazette de Clèves », c'est-à-dire Jean Manzon, rédacteur du Courrier du Bas-Rhin ${ }^{1}$. Ce journaliste y vante longuement les services qu'il rend à la Russie depuis le déclenchement de la guerre avec la Turquie, deux ans plus tôt, et demande les moyens d'être mieux informé à partir de SaintPétersbourg, mais aussi depuis la région du Danube et celle du Levant où opèrent les armées et la flotte russes :

\footnotetext{
${ }^{1}$ En abrégé CBR. La lettre que nous citons est insérée dans l'abondante correspondance de service adressée par D.A. Golitsyn au vice-chancelier Alexandre Mikhaïlovitch Golitsyn et conservée à Moscou, Archives russes d'État des actes anciens (RGADA), fonds 1263-Golitsyn, op. 1, n $1117-1124$ pour les années considérées ; le même fonds contient des minutes des lettres du prince A.M. Golitsyn à D.A. Golitsyn, principalement dans les dossiers $n^{\circ} 4137$ et suivants (référence abrégée : G- suivi du numéro du dossier et du folio) ; sauf indication contraire, les lettres citées sont adressées au vice-chancelier par D. A. Golitsyn ; les dates, que, sauf exception, nous indiquons telles qu'elles sont portées sur les lettres, sont celles du calendrier julien (orthodoxe), alors en retard de onze jours sur le calendrier grégorien utilisé en Occident. Sur cette correspondance, voir G. Dulac, avec la coll. de Ludmila Evdokimova, «Littérature et politique : la correspondance de Dmitri A. Golitsyn », Dix-huitième siècle, n²2, 1990, p. 369-402.
} 
Si Votre Excellence se fait lire quelquefois le Courier du Bas-Rhin, Elle aura remarqué que l'Ecrivain de cette feuille s'est imposé, depuis longtems, la tâche bien flatteuse pour lui, de mettre dans leur plus beau jour, dans leur jour véritable, les évenemens glorieux qui ont si particulierement distingué la Nation Russe, depuis deux ans. Non seulement je me suis attaché à ne laisser dans l'oubli, aucun des faits qui pouvoient contribuer à la gloire de la Russie ; mais j'ai eu l'attention la plus particuliere à démasquer les Ecrivains de feuilles périodiques, qui cherchoient par des doutes, ou par des assertions fausses, à obscurcir l'éclat des plus belles actions, et des conquêtes les plus importantes, des armées de Votre Illustre souveraine.

Faisant allusion à la cour de Versailles et à la guerre froide qui l'oppose à celle de Pétersbourg, il insiste sur les risques qu'il a pris :

[...] mon inclination pour la Nation Russe, m'a attiré des ennemis puissans que je ne nomerai pas, qui ont fait à mon sujet, dans un païs où je ne suis pas, qui même n'est pas celui de ma naissance, mais où l'on sait que j'ai demeuré, des perquisitions séveres, pour savoir mon nom, mes relations, qui m'ont suscité mille obstacles dans le débit de cette feuille qu'ils ont défendue chez eux, et qui me réserveroient à une vengeance plus cruelle, si je tombois jamais dans leurs mains.

Enfin il demande qu'on lui procure des correspondants particuliers, qui auraient ordre de lui faire parvenir leurs lettres par les moyens les plus rapides car, de manière bien sûr tout à fait désintéressée, il saura " louer les bonnes choses », les grands talents et les belles actions. Manzon entend en effet imposer l'idée qu'il n'est pas un journaliste ordinaire, puisqu'il possède le talent littéraire qui permet de mettre en valeur l'information :

Je ne demande que d'être bien instruit, d'être instruit promptement, et plus promptement que les autres, s'il étoit possible ; et je prends sur moi le soin de faire valoir les nouvelles qui me parviendront. [...] Les succès des Armées de S. M. Impériale seront sans doute annoncés au Public par les autres feuilles périodiques ; mais j'ose dire, qu'aucun des Ecrivains de ces feuilles, n'aura l'attention, ou la volonté, ou peut-être le talent de faire valoir ces succès autant qu'ils le meritent. Annoncer un fait sechement, c'est uniquement apprendre une nouvelle au Public : Mais l'annoncer avec chaleur, avec feu, avec éloquence, c'est faire une impression durable sur le Lecteur ; c'est le pénétrer de l'importance du fait qu'on lui annonce, et le lui rendre aussi intéressant qu'il nous le paroit à nous-mêmes. Pour parvenir à ce but, il faut de l'imagination, de l'art ; il faut posséder la Langue dans laquelle on écrit ; et surtout, il faut savoir faire quelque chose de plus qu'une gazette : qualités qui ne se trouvent gueres chez les gazetiers d'esprit et de profession.

Tout en assurant que la Russie n'a «aucune espece de besoin du secours des Gazettiers et des Nouvellistes », D. A. Golitsyn commente favorablement cette demande, en expliquant que le rédacteur du Courrier du Bas-Rhin «insinue de le gratifier soit par une petite pension tant que la guerre durera, soit par quelque petite somme une fois payée $»^{2}$. Il conservera des relations avec Manzon, lui fournira des documents à publier et à l'occasion saura lui «laver la tête », selon son expression ${ }^{3}$,

\footnotetext{
${ }^{2}$ Post-scriptum en clair d'une dépêche chiffrée datée du 27 avril 1770 (G-1117, 137v). En novembre 1770, le vice-chancelier annoncera l'envoi d'une lettre de change de 262 florins 10, soit 50 ducats, destinés à récompenser le journaliste (G-4210, 7v). Sur Mazon, voir la notice, par François Moureau, du Dictionnaire des journalistes, sous la dir. de Jean Sgard, Oxford, Voltaire Foundation, t. II, p. 676-679.

${ }^{3} 10$ janvier 1772 (G-1121, 10r).
} 
quand il lui arrivera de donner quelque article déplaisant pour la Russie : par exemple à propos de Rulhière et de son Histoire de la révolution de Russie, que l'auteur lit dans les salons de Paris et dont le journaliste a présenté une image flatteuse en novembre 1771, le jugeant " digne d'être comparé aux plus beaux morceaux de Salluste et de Tacite » (CBR, 1771, p. 731 $)^{4}$. D. A. Golitsyn, qui se fait une haute idée de l'importance de l'opinion publique en matière de politique, donne fréquemment des informations sur les gazettes, surtout hollandaises, et signale qu'il y fait insérer des communiqués ou des rectificatifs. Cependant c'est le Courrier du Bas-Rhin et dans une moindre mesure la Gazette des deux Ponts ${ }^{5}$ qui lui paraissent capables de se montrer favorables aux intérêts russes, cette dernière grâce surtout aux relations personnelles qu'il entretient avec le duc des Deux-Ponts, avec la comtesse de Forbach, son épouse morganatique, et sans doute aussi avec Jean Dubois-Fontanelle, le directeur de la gazette.

Je me suis donc proposé de croiser deux types d'informations : celles que fournit la correspondance de D.A. Golitsyn et celles qu'offrent ces deux gazettes. Ce travail reste cependant très limité, faute de dépouillements complets, surtout pour la Gazette des Deux Ponts, dont je n'ai examiné que l'année 1774. Par ailleurs, bien des questions d'interprétation ne recevront que des réponses hypothétiques ou resteront irrésolues, pour au moins deux raisons : on ne peut que soupçonner des influences multiples, ou diverses pressions politiques, qu'il est difficile de démêler ; et d'autre part les gazettes, parce qu'elles réagissent constamment les unes par rapport aux autres, forment un système complexe qu'il faudrait pouvoir analyser dans son entier à chaque moment. À cet égard, je me suis contenté de donner quelques exemples empruntés à la Gazette de France, qui offre le prototype de la plupart des thèmes anti-russes auxquels les autres périodiques s'efforcent de répondre quand ils s'engagent dans le parti opposé. Enfin il

\footnotetext{
${ }^{4}$ Depuis 1768 , Catherine II avait essayé d'obtenir de l'auteur qu'il supprime cet ouvrage qui relatait de manière parfois irrévérencieuse les événements qui lui avaient permis d'accéder au trône. Mais les interventions de son chargé d'affaires à Paris, Nikolaï Khotinski, puis de Diderot, avaient été infructueuses : voir à ce sujet ce que ce dernier écrit à Falconet, mais à l'intention de l'impératrice, en mai 1768 (Diderot, Correspondance, éd. Georges Roth et Jean Varloot, Paris, Minuit, 1955-1970, t. VIII, p. 32-33 et passim. Cependant l'affaire était toujours d'actualité : en juin 1771, D.A. Golitsyn avait transmis à Pétersbourg une longue lettre de Diderot qui racontait comment il venait de mystifier Rulhière et de discréditer son ouvrage dans la société parisienne (Georges Dulac, «Échec à Rulhlière, un récit inédit de Diderot rapporté par D. Golitsyn », Dix-huitième siècle, n 23, 1991, p. 212-222).

${ }^{5}$ Sur ces gazettes, voir les notices n ${ }^{\circ} 286$ et 507 du Dictionnaire des journaux 160o-1789, sous la dir. de Jean Sgard, Paris, Universitas, 1991, t. I, respectivement p. 301-305 (par François Moureau) et p. 463-464 (par Jacques Wagner) et, sur le Courier du Bas-Rhin, la thèse de Mathias Beermann, Zeitung zwischen Profit und Politik. Der Courrier du Bas-Rhin (1767-1810) (1996). Notons que concernant les deux périodiques jumeaux désignés couramment comme Gazette des Deux-Ponts, il semble régner une certaine confusion dans le Dictionnaire des journaux, puisque d'après la notice $n^{\circ} 578$, la gazette politique, qui a porté successivement plusieurs titres, semble n’avoir commencé qu'en 1778. Le témoignage de D. A. Golitsyn à ce sujet est très clair (18 janvier 1771, G-1119, 16v) : «On a etabli à DeuxPonts deux gazettes, dont une Litteraire et l'autre politique. Je les reçois regulierement. La premiere est trés interressante par la façon dont on y expose les nouveaux ouvrages et la seconde trés impartialle. On me prie de procurer un correspondant de Petersbourg [...]. S'il y avait même à Petersbourg quelcun qui voulut se charger de cette correspondance par interet, il pourrait faire ses propositions à $\mathrm{Mr}$ de Fontanelle aux Deux-Ponts. Toutes les conditions qui pourraient lui convenir seront reçues avec autant de plaisir que de reconnaissance. Comme j'ai de vrais amis à cette cour, je voudrais leur procurer cette satisfaction, independament du plaisir que j'aurai moi même de voir leur gazette politique ne donner que des nouvelles sures des evenemens du jour et des fruits des travaux de notre auguste Imperatrice ; et la Litteraire, faire connaitre à quel point les lettres sont cultivées en Russie sous Sa protection. »
} 
aurait fallu considérer, au-delà du contenu positif des gazettes, le vaste domaine des non-dits, qui ne sont pas moins significatifs, mais dont le repérage exigerait également une enquête bien plus étendue que la nôtre.

\section{Information et désinformation sur un sujet de premier plan}

Je ne rappellerai que très sommairement l'actualité politique très chargée de la période considérée, en ce qui concerne la Russie : la guerre diplomatique et de propagande qui depuis 1767 au moins oppose les cours de Versailles et de Pétersbourg, et se prolongera après la chute de Choiseul, en décembre 1770 ; les affaires de Pologne, depuis que Stanislas-Auguste Poniatowski a été élu roi en 1764 sous la pression des troupes russes ; la guerre russo-turque, déclenchée en octobre 1768 à l'instigation de la France, et le développement des confédérations polonaises, qui s'opposent à la fois au roi et aux Russes, avec le soutien de la France ; le spectaculaire passage de la flotte russe de la Baltique à la Méditerranée, qui aboutira à la destruction de la flotte ottomane à Tchesmé, en juillet 1770 ; le premier partage de la Pologne annoncé en 1772 et rendu effectif en 1773 ; la « révolution de Suède » qui en 1772 restaure l'absolutisme, au détriment de l'influence russe ; enfin l'insurrection de Pougatchev, en 1773-1774...

La correspondance de D. A. Golitsyn offre un tableau assez précis de la manière dont il considère les gazettes, du point de vue des intérêts russes. Quatre faits sont ainsi mis en valeur :

- la puissante campagne anti-russe menée par les agents de Versailles «qui sont partout ». Outre la Gazette de France, ce sont bien d'autres journaux et jusqu'à la Gazette d'Amsterdam qui diffusent parfois des informations tendancieuses et des fausses nouvelles qui tendent à ruiner le crédit de la Russie : les événements militaires, mais aussi la peste de Moscou en 1771, la révolte de Pougatchev, etc., fournissent nombre d'occasions pour de telles interventions, qui vont jusqu'à troubler certains diplomates russes, comme le note D. A. Golitsyn à propos de son collègue N. Khotinski, chargé d'affaires à Paris :

Quant à moi, [...] écrit D. A. Golitsyn le 29 mars 1774, j’y commence par dire, on dit cela, donc, cela n'est pas vrai ; car si cela etoit ainsi, on le diroit autrement. J'ai adopté la dessus le Sisteme de la Condamine, qui me le mandoit deux jours avant sa mort, et pretendoit s'en trouver aussi bien que celui qui depuis trente ans avoit ajouté deux Avé Maria à son rosaire. Voila ma regle en fait de nouvelles sur la Russie [...] des qu'il s'agit de la France. Il semble qu'on y a etabli une manufacture de faussetés et de mensonges ; et le peu de bons Ecrivains que la Cour ou le Ministere y protege, n'en sont plus employés qu'a ecrire contre nous. (G-1124, 43r-v)

- les moyens de pression dont dispose la cour de Versailles sur les journaux publiés hors de France, car elle pourra par exemple obtenir que la Gazette d'Utrecht soit suspendue pour avoir plaisanté aux dépens de la Dauphine :

La cour de Versailles s'en est formalisée et en a porté des plaintes demandant une punition exemplaire. Le magistrat d'Utrecht y a eu d'autant plus d'egard que leur ville ayant des fonds trés considerables en France, ils ont de grands menagemens pour elle. Le pauvre gazettier a eté severement reprimandé et de plus, on lui a deffendu pendant 6 semaines sa gazette, de maniere que vous n'en aurez point pendant tout ce tems là, et peut etre d'avantage, parceque on a fait demander à la Cour de Versailles si elle en etait contente, sinon, le tems sera 
prolongé. Le pauvre gazettier est ruiné de l'aventure. Jugez ce qu'on aurait fait si l'auteur du gazettier Cuirassé tombait entre leurs mains. (7 septembre 1771 (G-1120, 71v-72)

- les enjeux politiques importants de l'image de la Russie diffusée par la presse : pour financer la guerre, Pétersbourg a besoin d'emprunter sur la place d'Amsterdam, et ces négociations sont d'autant plus difficiles que le pays est jugé peu capable de supporter une guerre, et surtout instable, comme le souligne avec insistance D. A. Golitsyn en août 1770 :

[...] on nous prete difficilement de l'argent, tandis que l'angleterre, la France jusqu'à sa derniere crîse, l'autriche, la Saxe, $\&^{c}$. n'ont qu'a paraitre en desirer pour en avoir tant qu'elles veullent. Il faut donc qu'il y ait un vice radical dans tout ceci. Mais en voici la raison. [...] on ne leur otera jamais de la tête du peu de fond qu'on doit faire sur la Russie, uniquement à cause des revolutions frequentes qui y arrivent. Et ces revolutions etant toujours inattendues, inesperées, ils disent, que le Successeur de l'Empire de Russie n'a pour ainsi dire aucun rapport avec son predecesseur, et par là se croit dispensé de remplir les engagemens qu'il aura contracté.

Pour detruire cette idée, je ne vois qu'un moyen ; c'est celui d'y faire intervenir le Senat. Tant que nous ne parviendrons point a inspirer cette confiance dans les pais etrangers, nous ne pourrons jamais y emprunter des sommes tant soit peu considerables. (G-1118, 103)

- cette situation explique l'action soutenue menée en ce domaine par D. A. Golitsyn, qui travaille à encourager les journaux favorables à la Russie et s'efforce d'autre part de faire insérer des "vérités » dans les gazettes, surtout de Hollande, mais aussi de la région rhénane. Son réseau de relations étendu dans les milieux de la presse et de la « librairie » pouvait lui faciliter la tâche. Deux exemples : le ministre de Russie est très lié avec Marc-Michel Rey ${ }^{6}$, pour qui Manzon effectue des travaux de traduction ${ }^{7}$; quant à Dubois-Fontanelle, le directeur de la Gazette des Deux Ponts, qui à la demande de Cramer, à Genève, contribue en 1771 au lancement d'une réédition de l'Encyclopédie, il compte apparemment sur D. A. Golityn pour trouver des souscripteurs en Russie et leur consent des conditions avantageuses (23 avril 1771, G-1119, 113v).

Avant d'examiner comment évolue, dans ces conditions, le contenu des deux gazettes, j'apporterai quelques précisions à leur sujet.

Le Courrier du Bas-Rhin, qui paraît deux fois par semaine sur huit pages, est une des plus importantes gazettes francophones. Son directeur, Jean Manzon (1740-1798), originaire du Piémont, a peu séjourné en France. Ce journal, essentiellement politique, est publié à Clèves, en terre prussienne, soutenu par Frédéric II et soumis au contrôle d'un fonctionnaire prussien, qui à partir de 1769 dirige l'imprimerie qui le produit ${ }^{8}$. Cependant pendant la période qui nous intéresse, il y est peu question de Berlin et de la Prusse, au point que cette discrétion conduit parfois à s'interroger ${ }^{9}$. Ainsi pendant la

\footnotetext{
${ }^{6}$ George Dulac, «Littérature et politique : la correspondance de Dmitri A. Golitsyn », art. cit., p. 382, 384387.

${ }^{7}$ Dictionnaire des journalistes, notice « Manzon », op. cit., p. 676.

${ }^{8}$ Sur le Courrier et sa tutelle prussienne, voir Ute Van Runset: «La politique de Frédéric II et les gazettes : entre le Courrier du Bas-Rhin et la Gazette de Cologne », Gazettes et informations politiques sous l'Ancien Régime, éd. Henri Duranton et Pierre Rétat, avec une introduction de Keith M. Baker, Publications de l'Université de Saint-Etienne, 1999, p. 233-240: l'auteur envisage principalement l'attitude du Courrier à l'époque de la guerre de Succession de Bavière (1778-1779).

${ }^{9}$ Annie Riverra note à propos de l'année 1775 que le Courrier du Bas-Rhin, selon la volonté de Frédéric, reste « toujours presque muet sur la Prusse », ce qui contraste avec l'abondance des rubriques consacrées
} 
plus grande partie de 1772, quand se prépare le partage de la Pologne, le journaliste ne parle durant plusieurs mois que des «deux puissances impériales », la Russie et l'Autriche, comme si la Prusse n'était pas partie prenante: attitude qui répond vraisemblablement à la volonté du roi, alors que son frère, le prince Henri, s'était rendu à Pétersbourg pour préparer le partage dès les derniers mois de $1770^{10}$. Manzon se flatte d'avoir de nombreux correspondants et sa gazette est effectivement composée de lettres présentées comme d'origines très diverses. Il a des relations très suivies avec la Pologne : à partir de 1772, il rend divers services à Stanislas-Auguste, qui lui verse une pension et lui fait parfois passer des textes de sa main ${ }^{11}$.

La Gazette des Deux-Ponts est double, comme nous l'avons vu plus haut : DuboisFontanelle publie à partir d'avril 1770 la Gazette universelle de littérature, qu'on désigne souvent par le premier titre (en abrégé GDP-L), et à partir du mois suivant la Gazette des Deux-Ponts proprement dite, c'est-à-dire la gazette politique (GDP-P). Quelques mois plus tard, D. A. Golitsyn se targue d'avoir une certaine influence sur ces publications (18 janvier 1771, G-1119, 16r), mais il signalera aussi que leur attitude est très fluctuante : après avoir à ses débuts souvent reproduit la Gazette de France, la gazette politique est devenue plus impartiale, voire favorable à la Russie en 1771 (12 mars 1771, G-1119, 73r-v) ; mais trois ans plus tard il considère qu'on n'y écrit plus que « sous la dictée du ministère de France » (22 mars 1774, G-1124, 40r).

\section{Abondance et fluctuations du commentaire}

Dans le Courrier du Bas-Rhin, les informations sur la Russie et les événements auxquels elle est mêlée sont à certaines périodes très abondantes, surtout en 1769 et 1770, et souvent placées en tête des livraisons. On peut les ranger en quatre catégories, d'importance inégale.

En premier lieu, ce qui concerne l'actualité immédiate (la guerre russo-turque, les affaires de Pologne, etc.), informations rapportées à de multiples origines, Pétersbourg, Varsovie, Dantzig, la région du Danube, Constantinople, etc. Mais ces indications d'origine sont parfois ou souvent fictives : ainsi le 27 avril / 8 mai 1770, D.A. Golitsyn envoie au vice-chancelier un numéro du Courrier qui comporte, sous l'indication Dantzig, des extraits d'une lettre qu'un officier de l'armée du comte Petr Roumiantsev a adressée à un de ses amis, à Pétersbourg (CBR n ${ }^{\circ} 6$ du 5 mai 1770, p. 283-284). Cet officier relate les succès russes et s'en prend aux journalistes qui s'appliquent à les masquer ; et D.A. Golitsyn de signaler que le texte lui en a été fourni par le Collège des Affaires étrangères (G-1117, 113v). Cette lettre supposée, venue de Pétersbourg, y fait donc retour, une fois imprimée par Manzon, pour confirmation de cette opération de propagande. Le journaliste publie également de nombreux documents officiels russes,

\footnotetext{
à la Pologne («L'information vide, le discours des gazettes sur le premier partage de la Pologne », Gazettes et informations politiques sous l'Ancien Régime, op. cit., p. 313-324, ici p. 320 et 323. Le roi de Prusse, principal instigateur du partage, préférait agir en sous-main, comme le notait Jean Fabre (Stanislas-Auguste Poniatowski et l'Europe des Lumières, Paris, Ophrys, 1984, p. 246 et suiv.).

${ }^{10}$ Le 30 septembre 1772, le Courrier publie une longue déclaration de Frédéric II qui justifie la prise de possession de territoires jusque-là polonais (CBR, 1772, p. 656-658).

"Notice "Manzon", Dictionnaire des journalistes, op. cit., p. 677; Jean Fabre, Stanislas-Auguste Poniatowski et l'Europe des Lumières, op. cit., p. 475 (le rédacteur du Courrier du Bas-Rhin y est dénommé par erreur Mauzon).
} 
parfois très longs : par exemple en janvier 1769 une déclaration de Catherine II sur la protection des dissidents de Pologne (CBR 1769, p. 39-40) ; également des relations autorisées des victoires russes (ainsi le 15 août 1770 une lettre du comte Roumiantsev, CBR 1770, p. 515-516) ; en août 1773, un très long manifeste, publié en plusieurs fois, sur la rupture des négociations avec la Turquie (CBR 1773, p. 476-478, 484-486, 492-493). En dehors de ces documents officiels, les informations favorables à la Russie sont souvent répétées et amplifiées dans plusieurs numéros : ainsi la victoire navale de Tchesmé (juillet 1770) est brièvement annoncée le 4 septembre 1770, telle qu'elle est parvenue à la connaissance de D.A. Golitsyn, puis développée en trois récits successifs, de plus en plus détaillés, jusqu'à la fin octobre (CBR 1770, p. 574, 598-599, 618-621, 690691). D'autres thèmes de la propagande russe sont repris, souvent avec insistance, comme la satisfaction des Valaques et des Moldaves « nouvellement sujets de la Russie fort contents d'avoir changé de maître»(CBR 1770, p. 2), l'espoir des Grecs d'être délivrés du joug ottoman, ou «la fureur de persecuter \& de piller » qui anime les Confédérés dans un pays où « sous pretexte de zele pour la religion, on martirise les pauvres protestans » (CBR 1770, p. 149).

Le Courrier fournit d'autre part à ses lecteurs des informations politiques de fond, ou du moins qui ne sont pas liées à l'actualité immédiate. En 1770 et au début de 1771, ce sont principalement de longs extraits commentés du Nakaz de Catherine II (en six livraisons), puis de l'Antidote, sa fielleuse réfutation du Voyage en Sibérie de Chappe d'Auteroche : ces initiatives du journaliste sont très vraisemblablement commanditées par D. A. Golitsyn qui venait précisément de donner, chez M.-M. Rey, des rééditions de ces deux ouvrages ${ }^{12}$. Manzon les commente bien sûr très favorablement et met en valeur cette réflexion de l'auteur de l'Antidote qui en résume bien l'esprit : « avec un coup d'œil philosophique, [...] on verroit [que la Russie] est à peu près de niveau avec toutes les autres nations de l'Europe »(CBR 1770, p. 823). Quelquefois, cet effort de propagande prend un tour assez naï, par exemple quand, en août 1773 , le journaliste mentionne parmi les «établissements utiles faits par l'impératrice de Russie », les instructions qu'elle a données concernant le logement des paysans : alors que ceux-ci n'ont généralement " pour tout asile que des huttes si basses qu'on ne peut s'y tenir debout, enfoncées dans la terre, couvertes de boue, où l'on ne voit qu'un mauvais grabat pour le maître [...], S. M. I. a donné ordre de procurer à cette portion de ses sujets des habitations plus élevées, plus vastes, plus saines [...] ». Ainsi, par décision impériale, les paysans russes seront désormais confortablement logés (CBR 1773, p. 509).

Deux autres catégories d'informations occupent une place plus réduite dans le Courrier, mais reviennent cependant assez régulièrement. Elles concernent :

- les magnifiques festivités de la cour de Pétersbourg, qui sont souvent évoquées avec beaucoup de détails : par exemple la célébration de l'anniversaire de l'impératrice (CBR du 27 mai 1769, p. 331), la réception triomphale offerte à Pétersbourg au prince

\footnotetext{
${ }^{12}$ George Dulac, «Littérature et politique : la correspondance de Dmitri A. Golitsyn », art. cit., p. 384-385. Notons à ce propos que le 29 avril 1769 , Manzon avait rapporté que le prince Koslovski était venu à Ferney remettre à Voltaire, de la part de l'impératrice, « le code de loix que cette princesse a fait compiler pour son empire » (CBR 1769, p. 268) : cet ouvrage « signé de sa propre main » était en fait la version française de son Instruction aux députés (le Nakaz), qui venait d'être publiée à Pétersbourg. L'événement est relaté avec beaucoup de détails par Grimm dans sa Correspondance littéraire sous la date du 15 mars 1769, éd. Maurice Tourneux, Paris, Garnier, 1877-1882, t. VIII, p. 309-310.
} 
Henri de Prusse (CBR du 8 décembre 1770, p. 783-784), ou encore les honneurs rendus à Nikita Panine (CBR du 3 novembre 1773, p. 699-700), puis le mariage du grand-duc Paul (CBR du 17 novembre 1773, p. 733).

- les activités de l'Académie impériale des sciences, et parfois aussi de l'Académie des beaux-arts et de la Société libre d'économie : les voyages de Peter Simon Pallas en Sibérie et dans la région de la Caspienne sont par exemple commentés plusieurs fois (CBR 1773, p. 747-749, 775-776, etc.).

Outre le volume souvent étonnant des informations concernant la Russie, il faut considérer les prises de parti très marquées qui les accompagnent. Elles se glissent dans les lettres supposées venues de divers lieux, et d'autre part dans des commentaires éditoriaux datés du «Bas-Rhin» ou de Clèves. Dans le cas des correspondances présentées comme reçues de l'étranger, le «nous» du rédacteur donne une couleur d'authenticité aux commentaires, mais produit parfois des effets assez curieux, par exemple quand depuis Constantinople l'auteur de ces lettres s'indigne des atrocités commises aux dépens des Grecs par « les farouches Ottomans» (CBR 1770, p. 308), souligne les revers subis par les Turcs («nos troupes», CBR 1770, p. 62), ou tient des propos défaitistes sur les capacités militaires du pays (« nous ne comptons pas trop sur nos propres forces », CBR 1770, p. 204).

Les orientations générales du Courrier sont donc sans surprise, au moins en 17691770 : exaltation des succès des forces russes et de leur rôle protecteur (en Pologne), ou libérateur (en Orient) ; dépréciation de l'adversaire turc ; condamnation répétée des confédérés polonais, traités régulièrement de brigands et de fanatiques, condamnation à laquelle s'ajoute fréquemment des sarcasmes à l'adresse de la Gazette de Cologne : cette gazette catholique et ultramontaine, dirigée par un ex-jésuite, Jacques Dambrin, et protégée par le nonce apostolique de la ville ${ }^{13}$, prend en effet le parti des confédérés et s'emploie à déprécier les succès russes. On peut noter à ce propos que ces attaques dirigées contre les « révérends Pères » sont soit formulées par l'auteur du Courrier (par exemple CBR 1770, p. 293 ; 1771, p. 13 ; 1772, p. 830), soit insérées sous la forme d'un extrait de la Gazette de Saint-Pétersbourg, qui s'en est prise aux " absurdités que la gazette de Cologne continue de se permettre sur l'état de nos armées \& leurs opérations » (CBR 1770, p. 285-286). Observons enfin qu'en cette période d'entente cordiale entre Frédéric et Catherine II, bien des thèmes développés par le Courrier répondent aux intérêts de la Prusse autant que de la Russie : par exemple quand sont évoquées les persécutions subies par luthériens et réformés de la part des confédérés, ou lorsque le journaliste, au moment du dépeçage du royaume de Stanislas-Auguste, explique avec un admirable cynisme que « cette révolution importante \& nécessaire au bonheur de la Pologne" est également favorable à la paix en Europe, puisque désormais « regne la plus heureuse harmonie entre les diverses puissances » (CBR 1772, p. 832).

Un des aspects intéressants de ces prises de parti réside dans les procédés rhétoriques assez élaborés du journaliste. J'en retiendrai deux, parfois associés. Manzon recourt volontiers à la prédiction, voire à la prophétie : ainsi au début du conflit avec la Turquie, certainement pour s'opposer à la propagande française, il annonce que la Russie sera en mesure de financer la guerre, car elle aura toujours la faculté d'emprunter aux Anglais (CBR 1769, p. 183) ; l'année suivante, après les premiers succès

\footnotetext{
${ }^{13}$ Notice $n^{\circ} 506$ du Dictionnaire des journaux, op. cit., p. 462.
} 
russes, il annonce que "Constantinople va être resserré de toutes parts; et que la grande âme de Catherine II l'emportera sur la puissance ottomane, et sur celle du protecteur de ce superbe empire » [c'est-à-dire la France] (CBR 1770, p. 188). Sur de tels thèmes, l'amplification est souvent remarquable : dès le printemps 1770, le journaliste annonce que les Russes peuvent désormais marcher jusqu'à Constantinople et qu'on y redoute la destruction de l'empire. L'esprit général des commentaires de Manzon est bien résumé dans ces lignes d'un éditorial de janvier 1771, à propos de la guerre russoturque :

[...] nous verrons d'un côté une puissance abbatue, découragée, sans troupes \& sans alliés, ébranlée de toutes parts, prête à succomber \& à être ensevelie sous ses propres ruines : nous verrons de l'autre, une nation formidable par ses forces de terre \& de mer, enhardie par les succès les plus avantageux \& les plus soutenus, par son courage, par des alliés respectables \& puissants, animée peut-être par le désir secret de montrer une bonne fois ce qu'elle vaut, \& de punir le mépris aparent que des peuples policés dès longtems ont fait d'elle [...] (CBR 1771, p. 34)

Cependant le Courrier du Bas-Rhin est loin de se maintenir constamment à ce niveau et on y discerne d'importantes évolutions, ou plutôt des fluctuations, qui ne manquent pas d'intriguer. Ainsi, alors qu'en janvier 1771 le journal consacre encore de nombreuses pages à la Russie, dès le mois suivant les informations la concernant se font plus rares, plus brèves et plus neutres. Faut-il y voir l'effet d'autres influences, ou bien simplement le journaliste se voit-il obligé de répondre à la curiosité changeante de ses lecteurs ? Car désormais, pendant plusieurs mois, ce sont les nouvelles de France qui prennent largement le dessus, avec tout ce qui concerne la résistance des parlements et autres cours souveraines à leur destruction entreprise par le chancelier Maupeou.

En 1772, une évolution encore différente se dessine : ce qui concerne la Russie occupe désormais une meilleure place, mais presque uniquement à propos de la Pologne, dont les trois puissances préparent le partage. Des accents nouveaux apparaissent, sans doute dus aux libéralités de Stanislas-Auguste dont a bénéficié le journaliste : une compassion marquée pour les malheurs du pays, des éloges de son souverain, qui est par exemple disculpé par un « patriote polonais » d'avoir favorisé la confédération de Random formée par les dissidents, partisans de la Russie, qui sont à l'origine des désastres du pays ; et le roi, déclaré "sage et philosophe », est crédité de s'être opposé autant qu'il le pouvait aux excès commis par les troupes russes (CBR 1772, p. 488, 535-536). Des propos analogues ne seront pas rares au cours des années suivantes. On peut noter d'autre part qu'en 1772 un des principaux articles concernant la Russie rend compte d'une étude provenant d'une université allemande qui établit que la population du pays est si réduite, proportionnellement à sa superficie, qu'on ne peut que douter de «sa puissance extérieure »: on en conclut à la "supériorité immense de [la France] sur ce vaste empire », puisqu'elle est presque aussi peuplée (CBR 1772, p. 637-638). D. A. Golitsyn signale dans une lettre du 22 septembre / 3 octobre qu'un résumé de cette étude a été publié par la Gazette de France du 14 septembre, dont il reproduit un long extrait (G-1121, 222) : sous la date du 18 septembre, Manzon n'a fait que reprendre à peu près littéralement l'article de cette publication officielle française. Sur un autre plan, le journaliste fait de grands éloges de la « révolution de Suède », événement « désagréable » pour la Russie, mais « si nécessaire 
pour [le] royaume », note-t-il, parce qu'en restaurant l'autorité du souverain il doit éviter au pays des malheurs analogues à ceux qu'avait causés l'anarchie polonaise (CBR 1772, p. 665). La ligne politique du journal a donc clairement évolué, mais sous quelles influences ? Peut-on songer à y voir un effet indirect des tensions qui opposent à certains moments les puissances partageantes? C'est une pure hypothèse.

Une autre prise de position étonne encore davantage. En septembre 1772, une dépêche datée prétendument de la Vistule affirme que le ministère russe est embarrassé par l'issue de l'affaire polonaise, probablement parce que la cour de Pétersbourg n'avait jamais voulu le démembrement du pays ; et de conclure que les conséquences géopolitiques risquent d'en être désastreuses pour la Russie :

après les efforts incroiables que Pierre le Grand \& ses successeurs ont fait pour rendre la Russie une puissance d'Europe, [...] ; après que dans l'instruction donnée à la commission chargée de former un nouveau code de loix, on a fait un chapitre tout exprès pour établir que la Russie était une puissance d'Europe, il pourroit bien arriver par l'évenement, par le peu d'influence qu'elle pourra prendre désormais sur la Pologne \&c. qu'elle ne devint en effet qu'une puissance asiatique [...]. (CBR 1772, p. 684)

Je risquerai une hypothèse à ce propos : l'inspirateur de ce texte pourrait bien être D. A. Golitsyn lui-même, qui précisément dans les derniers mois de 1772 écrit en confidence à son cousin le vice-chancelier qu'à son avis le partage de la Pologne n'a pas été à l'avantage de la Russie, mais plutôt de l'Autriche, et surtout de la Prusse, dont elle a tout à craindre ${ }^{15}$.

À cette époque la ligne du Courrier du Bas-Rhin est donc peu claire, et surtout fluctuante. Les années suivantes, les articles favorables à la Russie ne seront pas rares, sans pourtant qu'on puisse y voir un nouveau retournement. C'est précisément au cours de ces années 1772-1774 que le journaliste met fréquemment en avant les difficultés de son métier : face à des événements embrouillés, dont il ne peut avoir souvent qu'une connaissance incertaine, d'autant plus qu'il est nécessairement tributaire de correspondants, qui ont eux-mêmes du mal à connaître la vérité ou peuvent se révéler partiaux, il lui arrive de se tromper, avoue-t-il. Et de conclure modestement un long développement sur ce thème, en tête d'un numéro d'août 1773 : «Ces tristes reflexions, il faut en convenir, n'éclaircissent pas les doutes du lecteur ; mais elles justifient les bevues, les contradictions, l'ignorance des nouvellistes [...]. Ah M. le chanoine d'Estampes, que n'étes-vous ici avec votre machine volante qui fait cent lieues dans 3 heures! La belle occasion pour en faire usage !» (CBR 1773, p. 505). Quoi qu'il en soit, le journaliste affirme à plusieurs reprises sa volonté d'impartialité, ce qui

\footnotetext{
${ }^{14}$ Ces vues correspondent à «l'idée, longtemps défendue [en Russie], qu'il valait mieux avoir une influence sur l'ensemble de la Pologne que d'en obtenir une partie. " Lucien Bély, Les Relations internationales en Europe (XVII ${ }^{e}$-XVIII ${ }^{e}$ siècles), Paris, PUF, 2007, p. 574-575.

${ }^{15}$ Ainsi dans une dépêche datée du 16 octobre 1772 (G-1121, 297-298v), D. A. Golitsyn écrit par exemple : « Lors de l'arrangement des trois cours sur le partage de la Pologne, on disait generallement qu'une des conditions du Traité signifiait positivement que celles de Vienne et de Berlin forceraient la porte à faire la paix avec nous aux conditions proposées au Congrés de Fokchani. Si la chose est vraie, rien de plus sage que ce partage [...]. Mais dans le cas contraire etait il de notre interet d'agrandir le Roi de Prusse sans etre sur qu'il ne contribue pas sous main à faire durer la guerre. [...] N'est il pas vrai que le Roi de Prusse est le Prince de l'Europe qui entend le mieux ses interets ? Est il moins constant encor que par la position des Etats respectifs, il est ennemi naturel de la Russie ? En ce cas est il rien de plus heureux pour lui que de nous voir nous epuiser par une guerre [...]. »
} 
paraît une prétention quelque peu exagérée. Mais il est vrai que si l'information est généralement tendancieuse, elle n'est pas totalement unilatérale, peut-être par souci de crédibilité. Cependant on peut considérer comme tout à fait exceptionnelle la publication intégrale, en mai 1769 , d'un long manifeste de la noblesse de Lituanie qui rejoint la confédération de Bar en dénonçant les traités, lois et constitutions imposés par la Russie «au gré de son ambition \& de son intérêt \& par les voies les plus violentes », la Russie dont on ne peut plus « douter que, sous les dehors d'une amitié promise, [elle] n'ait pour but de nous soumettre à sa domination, \& qu'elle ne cache les plus funestes intentions sous des témoignages de désintéressement \& d'humanité » (CBR 1769, p. 302). Quelques semaines plus tard, alors que le journal dénonce régulièrement « ces brigands qui sous le nom de confédérés, commettent des excès horribles »(CBR 1769, p. 4), et multiplie les commentaires favorables à la présence salutaire des troupes russes dans le pays, on y découvre une lettre présentée comme adressée depuis Varsovie à son rédacteur et dont le contenu très nuancé est quelque peu surprenant en pareil contexte :

[...] A propos de confédérés, j’ai pensé tout comme vous sur leur compte, lorsque je les jugeois de loin, \& que je ne les connoissois pas : Aujourd'hui que je les vois de plus près, $[$...] je suis convaincu qu'ils ne sont pas tous des brigands; \&, par intérêt pour la vérité, pour l'impartialité, que je sais que vous cherchez de bonne foi, je dois vous dire, qu'il seroit bon, que vous ne les appelassiez pas généralement de ce nom. Ce sont en grande partie, des fanatiques, des enthousiastes, des esprits égarés, mais de bonne foi dans leur erreur, qui s'imaginent, \& qui sont fondés en effet, d'après leurs principes, à s'imaginer, que leur religion \& leur liberté ont été lésées. (CBR 1769, p. 483)

Notons encore l'insertion, en janvier 1770, d'une lettre datée de Varsovie qui dénonce vigoureusement la partialité, pour ou contre, des journalistes à l'égard des confédérés, selon la religion qu'ils professent, et leur incompréhension des ressorts profonds des événements en cours :

Je puis vous assurer, écrit ce correspondant, que vous étes très mal informé si vous vous en rapportez à leurs rélations ou absolument fausses, ou tronquées, ou déguisées. Il regne parmi les nouvellistes de vos cantons, une partialité, en vérité très indigne d'une personne qui fait metier de dire le pour \& le contre ; et qui ne doit jamais paraître pencher en faveur de l'un ou de l'autre parti. (CBR 1770, p. 55).

De telles interventions ne se reproduiront guère au cours des années suivantes. Et malgré certains propos plus nuancés insérés ici ou là, et certaines fluctuations que nous avons notées, dans l'ensemble la position du journal restera ouvertement favorable aux intérêts russes, et donc opposée à ceux de Versailles. C'est peut-être ce qui explique les graves entraves à sa diffusion, imputées notamment à « l'esprit de parti », que signale un long communiqué placé à la fin du dernier numéro de 1773. Et il est significatif qu'en promettant « de redoubler de soins et de dépenses » pour satisfaire le public, le journal annonce qu'il vient « de s'assurer des correspondances fort étendues \& fort exactes » sur le théâtre des opérations entre la Russie et la Turquie (CBR 1773, p. 834). En avril de l'année suivante, son correspondant parisien, Jean-Baptiste Plumex, sera arrêté par la police ${ }^{16}$...

\footnotetext{
${ }^{16}$ Dictionnaire des journaux, « Courrier du Bas-Rhin », op. cit., p. 301.
} 
À partir de 1774 cependant, la situation internationale a suffisamment évolué pour que la position du journal s'en ressente : les succès de la Russie, en Pologne et dans le conflit avec la Turquie, se sont affirmés, si bien que la cour de Versailles a dû en prendre son parti et envisager un rapprochement avec l'impératrice. Les prises de position du Courrier sont alors moins tranchées, et même les Turcs ont parfois droit à des égards, comme lorsque le journal publie la biographie d'un pacha, un «brave musulman », ou fait l'éloge du sultan défunt, Mustapha, " prince humain, pacifique, aimant sa famille [...] »(CBR 1774, p. 70 et 145). Le traitement des informations concernant la révolte de Pougatchev témoigne de cette attitude plus réservée, dont l'interprétation est cependant parfois délicate : dans l'ensemble, le journal s'en tient à l'explication formulée par Catherine II dans ses manifestes, qui attribuent les troubles à des bandes de brigands. Les succès des opérations de répression sont régulièrement signalés, mais leur répétition même, au fil des mois, et l'annonce des effectifs des troupes envoyées sur place rendent peu crédible la thèse officielle, que Manzon semble cependant reprendre à son compte lorsqu'il reproduit une lettre reçue de Pétersbourg par le prince D. A. Golitsyn, où il n'est question que de l'anéantissement de « voleurs de grands chemins » (CBR 1774, p. 164) : bientôt le journaliste préfèrera proposer une analyse de l'habileté tactique de Pougatchev (CBR 1774, p. 302-303). Cependant le trait le plus significatif de l'attitude du journal, surtout à partir de mai-juin 1774, est peutêtre la quasi-disparition du sujet pendant de longues périodes: des semaines se succèdent sans qu'il soit question de Pougatchev, si bien que la rébellion est reléguée à l'arrière-plan, par une sorte de non-dit qui contraste avec l'attitude de gazettes moins favorables à la Russie.

À cette époque, bien que D. A. Golitsyn soit parfois mentionné comme source des informations qui parviennent à la gazette ${ }^{17}$, il semble avoir moins de prise sur son contenu, comme le prouve un petit épisode qui concerne Diderot : en signalant le 16 avril le passage récent du philosophe à Hambourg, lors de son retour de Pétersbourg à La Haye, le journal ajoute qu'« on ne doute pas que ce métaphisicien célebre n'ait justifié la bonne opinion qu'on avoit de lui dans une cour dont il a reçu les plus grands bienfaits, sur la seule réputation de ses travaux, \& à la recommandation de l'ambassadeur de Russie résidant alors à Paris ", formulation qui laisse deviner que ce commentaire est dû à l'intervention de D. A. Golitsyn (CBR 1774, p. 246) ; mais le 7 mai la gazette annonce que « le célèbre $\mathrm{M}$. Didérot a eu le malheur d'encourir la disgrâce de l'impératrice, \& qu'on ne lui a donné que peu d'heures pour sortir de la capitale » (CBR 1774, p. 295). Comme le signale D. A. Golitsyn dans une lettre indignée qu'il adresse à Pétersbourg le 13 mai (G-1124, 77-78v), la fausse nouvelle de l'expulsion de Diderot est reprise de la Gazette d'Altona du 29 avril: elle y avait été insérée très vraisemblablement à l'instigation de Frédéric II qui, après avoir beaucoup fait quelques mois plus tôt pour discréditer Diderot, par l'intermédiaire de Formey, auprès de l'Académie des sciences et de la cour de Pétersbourg, s'était offusqué que le philosophe refusât obstinément de passer par Berlin. D. A. Golitsyn fera insérer un rectificatif dans la Gazette de Leyde, seule en Hollande à reprendre l'«information», mais aucun démenti ne paraitra dans le Courrier du Bas-Rhin.

\footnotetext{
${ }^{17}$ Par exemple dans le numéro du 12 mars, à propos des bandes anéanties par le général Bibikov (CBR 1774, p. 164), ou le 15 octobre au sujet de l'accord de paix que le nouveau grand-vizir a confirmé (CBR 1774, p. 657).
} 
En 1774, Manzon est cependant sous l'influence d'un autre diplomate russe, Otto von Stackelberg, ministre plénipotentiaire à Varsovie et véritable proconsul auprès du roi de Pologne, lui-même en relations étroites avec le directeur du Courrier qu'il pensionne, comme on l'a vu ${ }^{18}$. Cette situation explique que la Russie soit très présente dans les informations particulièrement abondantes que le journaliste fournit sur les affaires polonaises, mais surtout qu'il se trouve soumis à des pressions contradictoires : d'où semble-t-il certaines incohérences d'autant mieux assumées qu'il feint de les ignorer. Ainsi, pour ne citer qu'un exemple, alors que les puissances partageantes, représentées par Stackelberg, non contentes d'avoir amputé la Pologne de plus d'un quart de son territoire avaient, sous la menace de représailles qu'exerceraient les troupes russes sur la population, contraint son souverain à accepter la tutelle d'un Conseil permanent et la réduction des prérogatives royales, le Courrier du Bas-Rhin proteste quand la Délégation au service des puissances fait brûler par la main du bourreau et interdire la Gazette de Leyde qui avait critiqué ces mesures (CBR 1774, p. 593, 619, etc. $)^{19}$; il s'apitoie volontiers sur le sort d'un roi «malheureux \& dans le dépouillement "; il publie à sa demande une lettre pathétique qu'il a adressée à Stackelberg le 11 décembre précédent («Vous venez d'être l'instrument du cruel sacrifice dont je suis la victime innocente. Vous avez vu toute l'amertume de ma peine [...] » (CBR 1774, p. 547-548). Mais dans le même temps, il ne cesse de célébrer la grandeur, la générosité et l'équité de Catherine II, « cette souveraine magnanime » qui récompense ses généraux en leur offrant quelques dizaines de villages « dans la partie de la Pologne qu'elle s'est appropriée »(CBR 1774, p. 639); et qui, dans son admirable « libéralité », multiplie les actions qui « semblent porter l'empreinte de la grandeur de son âme », au point d'avoir voulu «dédommager de quelque manière » le roi de Pologne en lui adressant une lettre de change de 250 ooo roubles... (CBR 1774, p. 645). Car il est douteux qu'on puisse voir de l'ironie dans de telles pages.

\section{Les postures contrastées de la Gazette des Deux-Ponts}

Le journalisme, tel que le pratiquent les gazettes jumelles des Deux-Ponts, se situe dans l'ensemble à un autre niveau que ce qu'on observe dans le Courrier du Bas-Rhin : la partialité, l'intention de discréditer y sont certes souvent évidentes, et à lui seul l'examen de l'année 1774 confirme l'appréciation de D. A. Golitsyn sur l'influence déterminante que la cour de Versailles exerce sur la gazette politique à cette époque, aux dépens des intérêts russes. Cependant le jeu de l'information orientée s'y pratique avec plus de finesse et la propagande épargne une part notable du contenu rédactionnel. Ainsi, concernant la Russie, les deux gazettes, la politique comme la littéraire, portent une attention soutenue et bienveillante aux informations qui relèvent des domaines culturel et scientifique, y compris quand elles sont associées à la personne de l'impératrice, si bien que cette partie contraste fortement avec la manière souvent ironique, voire sarcastique, dont sont présentées les nouvelles proprement politiques : ainsi on loue l'attention qu'a désormais Catherine II de faire assister son fils

\footnotetext{
${ }^{18}$ D'après des documents conservés à Varsovie, Archives d'actes anciens (AGAD), fonds Popiel et mentionnés dans la notice «Manzon » du Dictionnaire des journalistes, op. cit., p. 678, Manzon a reçu cette année-là des recommandations à la fois de Stanislas-Auguste et de Stackelberg.

${ }_{19}$ Stanislas-Auguste avait un agent actif auprès de la Gazette de Leyde : sur cet épisode voir J. Fabre, Stanislas-Auguste Poniatowski et l'Europe des Lumières, op. cit., p. 475.
} 
aux différents conseils, pour le former à l'art de régner (GDP-P 1774, p. 289) ; on signale les institutions qu'elle a fondées à Pise pour éduquer des jeunes Grecs et pour assurer l'entretien de filles et de femmes du Levant (GDP-P 1774, p. 68). D'assez nombreuses informations sont apportées sur l'Académie impériale des sciences et celle des beauxarts, et leur composition est expliquée en détail (GDP-P 1774, p. 26). Les publications de Jacob Staehlin sur les découvertes géographiques des Russes dans l'« Archipel du Nord» (le Kamtchatka) et celles de Gerhard Müller sur l'histoire des premières tentatives d'explorations de cette région sont assez longuement évoquées dans la gazette politique (GDP-P 1774, p. 142-143, 166-167), et cette série se conclut sur un éloge de Catherine II, qui a favorisé les explorations annonçant l'ouverture d'une nouvelle route vers l'Amérique. Quant à la gazette littéraire, elle fait également une assez large place aux informations concernant la Russie, telles que le compte rendu des mémoires de l'Académie impériale des sciences, les Novi Commentarii (GDP-L 1773, p. 125) et celui d'une traduction du Journal de Pierre le Grand publié à Berlin (GDP-L 1773, p. 329).

Sur le terrain politique, il en va tout à fait différemment, bien que le journal n'adopte nullement un ton uniformément hostile. Les nouvelles des opérations militaires sur le Danube sont présentées sous un jour peu favorables aux Russes, et il en est de même des affaires polonaises : en février et mars 1774 la gazette politique signale par exemple les espoirs que suscitent chez les Polonais les troubles intérieurs que connaît la Russie et elle met en valeur le manifeste de la nouvelle confédération de Bar, qui veut annuler tout ce qui s'est fait par la volonté des puissances étrangères. Concernant l'insurrection de Pougatchev, le journal annonce en février que « les affaires intérieures de la Russie semblent devenir tous les jours plus critiques » et que «les révoltés se rendent à chaque instant plus formidables» (GDP-P 1774, p. 116). L'ironie est fréquente à propos de l'extrême discrétion, voire la rétention systématique de l'information pratiquée à ce propos dans la capitale russe; on se moque des euphémismes employés par la gazette française de Pétersbourg, qui qualifie les troubles de simples « inconvénients » (GDP-P 1774, p. 89). La Gazette des Deux-Ponts se fonde notamment sur les correspondances reçues par des marchands de Dantzig et de Hambourg pour insister sur la gravité de ces événements et les succès remportés par les rebelles. Pour s'opposer à la propagande russe qui présente Pougatchev comme un « imbécile », et probablement sur le modèle de la Gazette de France, elle lui prête une grande habileté tactique et en fait une sorte de héros : "cet homme aujourd'hui fameux [...] n'est pas un cosaque du commun ; mais d'une des premières familles de sa nation », il a été page de l'impératrice Elisabeth, a étudié à Berlin, puis a servi dans les troupes prussiennes, etc. «Ce roman de Pugatschew en vaut bien un autre, commente la gazette ; il est même appuyé par le manifeste de l'impératrice [...] » (GDP-P 1774, p. 195) : on notera l'habileté, ou la duplicité, du mode de présentation adopté par le journaliste, qui accrédite d'une certaine manière l'information, tout en prenant ses distances avec elle (c'est un roman...). Même souci de contredire, à propos de la Pologne, un des thèmes de la propagande russe, la protection des dissidents (les orthodoxes) au nom de la tolérance : dans les régions annexées, ces dissidents sont devenus à leur tour les persécuteurs de ceux qui sont d'une autre religion, « spectacle digne du $\mathrm{X}^{\mathrm{e}}$ siècle », ajoute la gazette (GDP-P 1774, p. 248).

\section{Conclusion}


Pour conclure, je rappellerai les quelques constatations qui me paraissent les plus importantes. Mais je voudrais aussi suggérer que bien des points resteraient à approfondir et à nuancer, bien mieux que je n'ai pu le faire.

Il est clair tout d'abord que la Russie est au début des années 1770 un sujet de tout premier plan pour ces gazettes politiques : même dans les moments où celle des DeuxPonts ne lui est guère favorable, elle place fréquemment les dépêches de Pétersbourg en première page, et toutes ne tendent pas à dénigrer, loin de là.

Il faut en effet souligner que les postures d'impartialité ont toujours leur place dans la rédaction de ces journaux, même si le déséquilibre entre informations ou commentaires pour et contre est le plus souvent patent. Peut-être même ne s'agit-il pas seulement de postures destinées à répondre aux exigences du public, mais parfois d'une certaine conscience de la dignité du métier de journaliste : bien que Manzon vende ses services, c'est l'impression que peut donner sa défense véhémente de la Gazette de Leyde, sanctionnée pour avoir critiqué les opérations qui ont suivi le partage. Mais il est vrai qu'en agissant ainsi, il répondait vraisemblablement aux vœux de Stanislas-Auguste...

Comme nous l'avons vu, le Courrier et la Gazette des deux Ponts ont en commun une certaine instabilité de leur orientation politique. Mais il est délicat d'en rendre compte exactement, et surtout de l'expliquer. Ainsi concernant les positions pour le moins ambigües qu'affiche le Courrier du Bas-Rhin en 1774, à propos de la Pologne dans ses rapports avec la Russie, on pourrait envisager plusieurs explications pour compléter celle que nous avons proposée : Frédéric II ne trouvait-il pas son compte à faire rejaillir sur le représentant de la Russie la noirceur de certaines opérations? Le journaliste n'était-il pas d'autre part tenté de tenir compte d'une certaine évolution de l'opinion publique, en Europe, après le partage ? Ce ne sont là que des hypothèses, mais elles présentent l'avantage de suggérer la complexité des conditions qui déterminent à un moment donné le discours journalistique. 